\title{
Image Retargeting with Protection of Object Arrangement
}

\author{
Kazu MISHIBA $^{\dagger \text { a) }}$ and Takeshi YOSHITOME ${ }^{\dagger}$, Members
}

SUMMARY The relative arrangement, such as relative positions and orientations among objects, can play an important role in expressing the situation such as sports games and race scenes. In this paper, we propose a retargeting method that allows maintaining the relative arrangement. Our proposed retargeting method is based on a warping method which finds an optimal transformation by solving an energy minimization problem. To achieve protection of object arrangement, we introduce an energy that enforces all the objects and the relative positions among these objects to be transformed by the same transformation in the retargeting process. In addition, our method imposes the following three types of conditions in order to obtain more satisfactory results: protection of important regions, avoiding extreme deformation, and cropping with preservation of the balance of visual importance. Experimental results demonstrate that our proposed method maintains the relative arrangement while protecting important regions.

key words: content-aware image retargeting, warping method, image resize, object arrangement

\section{Introduction}

As the diversity of electronic display devices increases (e.g., smart phones, notebooks, tablets, and HDTV), it is increasingly important that visual media display appropriately on these devices. Because the same image needs to be displayed with different resolutions on different devices, image resizing plays an important role in appropriate display. The classical resizing methods of homogeneous scaling and cropping have the drawbacks of distorting the entire image and discarding important parts of the image, respectively.

Content-aware image retargeting [1], [2] has recently been developed to overcome these limitations. Several retargeting methods have been proposed, including seam carving [3], [4] and image warping [5], [6]. The key concept of these techniques is to resize images to an arbitrary resolution while protecting visually important regions from distortion. Seam carving changes the size of a source image by iteratively carving out or inserting paths of pixels, which is called seam. A seam is selected to pass through less important regions to keep important regions unchanged. Warping methods place a mesh onto an image and then deform the mesh by solving an optimization problem. Many methods use a regular grid mesh and a few methods [6] use an irregular triangle mesh. To reduce distortions, several content-

Manuscript received December 3, 2013.

Manuscript revised February 19, 2014.

${ }^{\dagger}$ The authors are with the Department of Electrical and Electronic Engineering, Tottori University, Tottori-shi, 680-8550 Japan.

a) E-mail: mishiba@ele.tottori-u.ac.jp DOI: 10.1587/transinf.E97.D.1583 aware retargeting methods combine with classical resizing operators: a combination of seam carving with scaling [7], a combination of seam carving with scaling and cropping [8], and a combination of warping with cropping [9].

Many previous studies have focused only on protection of the shape of visually important regions. Therefore, conventional methods often shrink or enlarge unimportant regions among objects, resulting in the change of the distance among these objects. Figure 1(b) is an example of the reduction of the image width using a conventional method. Although the retargeting result may be satisfactory to a viewer, it can be unsatisfactory to a photographer if one considers that the distance between the main objects (horses) has a significant meaning in the image. The distance among objects can play an important role in expressing the situation such as sports games and race scenes like Fig. 1 (a). Thus, the change of a relative arrangement, such as relative positions and orientations among objects, can distort the intended content. In this paper, we propose a retargeting method that allows maintaining the relative arrangement among objects.

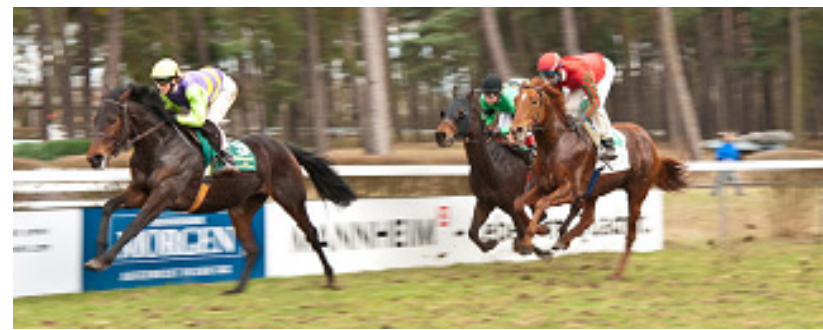

(a)

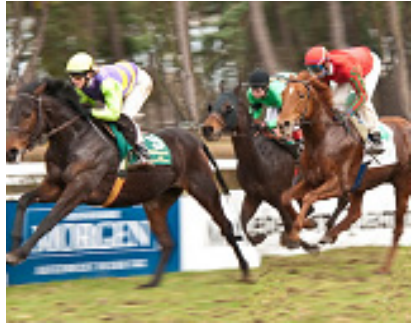

(b)

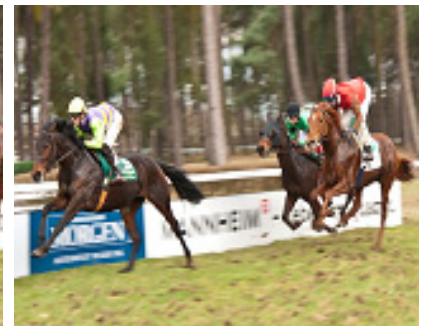

(c)
Fig. 1 The relative distance between the horses on the source image (a) is changed in the retargeting image (b) using seam carving [4]. The retargeting image (c) using our method maintains the relative distance. 


\section{Proposed Method}

Our proposed retargeting method is based on a warping method. In our warping method, a mesh is projected onto the image and then deformed to a target size to satisfy desired conditions as much as possible by solving an energy minimization problem. Our proposed method has four conditions to be satisfied.

The main condition is to achieve protection of the relative arrangement among objects on an image. In this paper, the relative arrangement between object $\omega_{i}$ and object $\omega_{j}$ is defined by two parameters $\left(\theta_{i, j}, r_{i, j}\right): \theta_{i, j}$ is the relative direction between the reference orientation of $\omega_{i}$ and the direction of $\omega_{j}$ from $\omega_{i}$, and $r_{i, j}$ is the ratio of the distance $l_{i, j}$ between $\omega_{i}$ and $\omega_{j}$ relative to the size $h_{i}$ of $\omega_{i}$, i.e., $r_{i, j}=h_{i} / l_{i, j}$ (see Fig. 2).

While regions among objects are unnecessary parts in conventional methods, they are important parts in our method for protection of the relative arrangement. Thus, our approach requires more space than conventional methods when an image size is reduced. Our warping method incorporates cropping to allocate space to objects and regions among them. Cropping can discard unnecessary regions, resulting in allocation of more space for necessary regions. To achieve better cropping, our method imposes the condition for preservation of the balance of visual importance during cropping (see Fig. 3).

Our proposed method introduces other two conditions to produce more satisfactory results. The first condition is that the shape of important regions is protected. The second condition is that extreme deformation is avoided.

The rest of this section is organized as follows. In Sect. 2.1, we formulate a retargeting problem as an energy minimization problem. In Sect. 2.2, the energy definitions are discussed. Section 2.3 describes the implementation of our proposed method.

\subsection{Problem Formulation}

An initial mesh $\mathbf{M}=\{\mathbf{V}, \mathbf{F}\}$ is represented as a set containing two components. $\mathbf{V}=\left\{\boldsymbol{v}_{i}\right\}_{i=1}^{\alpha}$ is a set of vertex positions where $\alpha$ is the number of vertices and $\boldsymbol{v}_{i}=\left[v_{i, x}, v_{i, y}\right]^{T}$ is a vector consisting of $\mathrm{x}$ and $\mathrm{y}$ coordinate values. $\mathbf{F}=\left\{f_{i}\right\}_{i=1}^{\beta}$ is

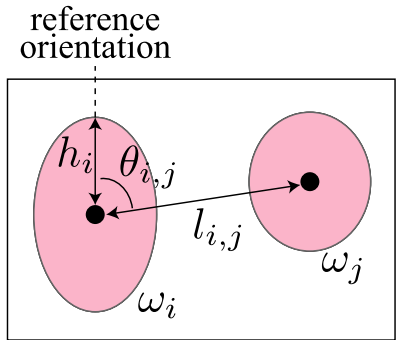

Source image

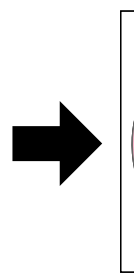

Retargeting image
Fig. 2 Example of the protection of object arrangement. a set of faces where $\beta$ is the number of faces. Our method can use both triangle and quadrilateral mesh. Let us denote an element after retargeting by adding a prime mark, e.g., $\boldsymbol{v}_{i}^{\prime}$ indicates a vertex position after retargeting. The transformation of an image of size $W \times H$ to $W^{\prime} \times H^{\prime}$ is a problem of finding optimal vertex positions $\mathbf{V}_{\text {opt }}^{\prime}$ by solving an energy minimization problem.

The energy minimization problem consists of energy functions $E$ and hard constraints. $E$ increases less as the deformed mesh better satisfies a desired condition. The optimal mesh transformation is expressed as

$$
\mathbf{V}_{o p t}^{\prime}=\underset{\mathbf{V}^{\prime}}{\arg \min } \sum_{z \in Z} \lambda_{z} E_{z}
$$

subject to hard constraints. Here $Z$ is a set of desired conditions, $E_{z}$ is an energy function for condition $z$ and $\lambda_{z}$ is a weight parameter for $E_{z}$. Using a larger $\lambda_{z}$ enhances the corresponding condition.

A retargeting image is obtained by extracting the pixels on the target range from zero to $W^{\prime}$ in width and zero to $H^{\prime}$ in height of the transformed mesh.

\subsection{Energy Definition}

We use the following four conditions to be satisfied in the retargeting process:

- maintaining the relative arrangement among objects,

- protection of important regions,

- avoiding extreme deformation,

- cropping with preservation of the balance of visual importance.

This subsection describes the following four energies corresponding to the above conditions: arrangement energy, rigid energy, smoothing energy, and cropping energy,

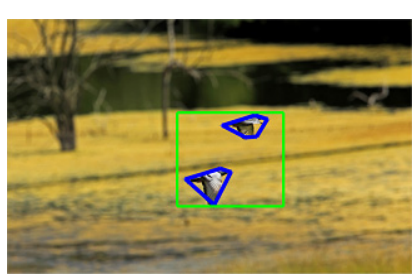

(a)

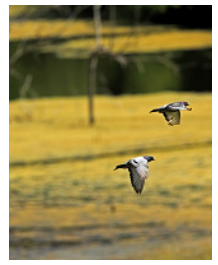

(c)

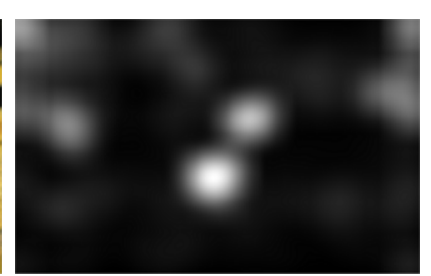

(b)

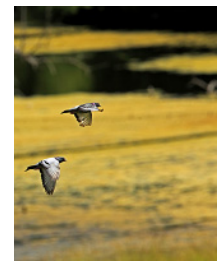

(d)

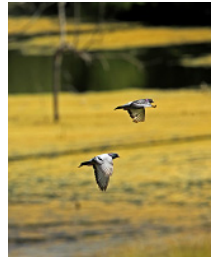

(e)
Fig. 3 Cropping with preservation of the balance of image importance. (a) Source image with selected regions (blue) and a rectangular range not to be discarded (green). (b) Image importance. (c), (d) and (e) three types of cropping. The retargeting result (e) preserves the importance centroid normalized by the image size. 
Arrangement energy. First, we describe which transformation maintains the relative arrangement among objects. Let us take the case of two objects, $\omega_{i}$ and $\omega_{j}$, as an example. Let us represent the position of $\omega_{i}$ by the centroid $\boldsymbol{g}_{\omega_{i}}$ and denote the relative position of $\omega_{j}$ relative to $\omega_{i}$ by $\boldsymbol{g}_{\omega_{i}, \omega_{j}}=\boldsymbol{g}_{\omega_{j}}-\boldsymbol{g}_{\omega_{i}}$. When $\omega_{i}$ is transformed by an affine transformation $T$ that rotates the reference orientation and scales the size of $\omega_{i}$, the relative position $\boldsymbol{g}_{\omega_{i}, \omega_{j}}$ should be transformed by $T$ to maintain the relative arrangement $\left(\theta_{i, j}\right.$, $\left.r_{i, j}\right)$. The same holds for $\omega_{j}$. Thus, we can see that all the objects and all the relative positions among objects should be transformed by the same transformation $T$ to maintain the relative arrangement among objects. Figure 2 shows an example of the protection of relative arrangement. In addition, $T$ should be a transformation that maintains the aspect ratios of objects to avoid distortion. Thus, a similarity transformation is used as $T$.

Next, we define the transformation and the centroid of an object to formulate the arrangement energy. They can be expressed using faces because an object is on faces of a mesh. The transformation of an object can be approximated by the transformation of the faces where the object is on. The $n$ edge vectors constituting the face $f$ are deformed from $\left\{\boldsymbol{e}_{f, i} \mid i=1, \ldots, n\right\}$ to $\left\{\boldsymbol{e}_{f, i}^{\prime} \mid i=1, \ldots, n\right\}$ in the retargeting process. Here, $\boldsymbol{e}_{f, i}=\boldsymbol{v}_{f, j}-\boldsymbol{v}_{f, i}$ is the $i$-th edge vector of size 2 constituting the face $f$, where $\boldsymbol{v}_{f, i}$ is a vertex of $f$ and $\boldsymbol{v}_{f, j}$ is the next vertex of $f$ in a clockwise order. The transformation of the face can be computed as the linear transformation of the mapping from before to after retargeting:

$$
\boldsymbol{J}_{f}=\left[\boldsymbol{e}_{f, 1}^{\prime}, \ldots, \boldsymbol{e}_{f, n}^{\prime}\right]\left[\boldsymbol{e}_{f, 1}, \ldots, \boldsymbol{e}_{f, n}\right]^{+}
$$

where $[\cdot]^{+}$is an operator that calculates $\boldsymbol{A}^{+}=\left(\boldsymbol{A}^{T} \boldsymbol{A}\right)^{-1} \boldsymbol{A}^{T}$ for matrix $\boldsymbol{A}$. This mapping is a linear approximation for a quadrilateral face. The centroid of an object can be approximated by the weighted average of the centroids of the faces where the object is on. The centroid of the face $f$ can be calculated by

$$
\boldsymbol{g}_{f}=\frac{1}{|\mathbf{V}(f)|} \sum_{\boldsymbol{v} \in \mathbf{V}(f)} \boldsymbol{v}
$$

where $\mathbf{V}(f)$ is a set of the vertices constituting the face $f$ and $|\mathbf{V}(f)|$ is the number of elements in $\mathbf{V}(f)$. The centroid of an object can be expressed as

$$
\boldsymbol{g}_{\omega}=\frac{1}{\sum_{f \in \mathbf{F}(\omega)} a_{f}} \sum_{f \in \mathbf{F}(\omega)} a_{f} \boldsymbol{g}_{f}
$$

where $a_{f}$ is an area of $f$. The centroid of an object after retargeting is approximately calculated using $a_{f}$ instead of $a_{f}^{\prime}$.

Finally, we define the arrangement energy $E_{a}$ for maintaining the relative arrangement among objects. From above discussion, we can see that the following two conditions should be satisfied to maintain the relative arrangement. The first condition is that all the faces where the objects are on are transformed by the same transformation $T$. The second condition is that all the relative positions among objects are transformed by the same $T$. Thus, $E_{a}$ can be expressed as the sum of two energies corresponding to these conditions.

The first condition is expressed as the energy

$$
E_{a 1}=\sum_{\omega \in \Omega} \sum_{f \in \mathbf{F}(\omega)} a_{f}\left\|\boldsymbol{T}_{\Omega}-J_{f}\right\|_{F}^{2}
$$

where $\Omega$ is a set of all objects, $F(\omega)$ is a set of the faces where the object $\omega$ is on, $\|\cdot\|_{F}$ is the Frobenius norm of a matrix, and $\boldsymbol{T}_{\Omega}$ is a similarity transformation:

$$
\boldsymbol{T}_{\Omega}=\left[\begin{array}{cc}
c & -d \\
d & c
\end{array}\right] .
$$

$E_{a 1}$ increases more as the transformation of a face is further deformed from $T_{\Omega}$.

The second condition is expressed as the energy

$$
E_{a 2}=\sum_{\left(\omega_{i}, \omega_{j}\right) \in \Phi_{\Omega}} l_{i, j}\left\|\boldsymbol{T}_{\Omega} \boldsymbol{g}_{\omega_{i}, \omega_{j}}-\boldsymbol{g}_{\omega_{i}, \omega_{j}}^{\prime}\right\|_{2}^{2}
$$

where $l_{i, j}=\left\|\boldsymbol{g}_{\omega_{i}, \omega_{j}}\right\|_{2}$ and $\Phi_{\Omega}=\left\{\left(\omega_{i}, \omega_{j}\right) \in \Omega \times \Omega \mid \omega_{i} \neq \omega_{j}\right\}$ is a set of all the possible ordered pairs of all objects. $E_{a 2}$ increases more as the transformation of the relative position $\boldsymbol{g}_{\omega_{i}, \omega_{j}}$ is further deformed from $T_{\Omega}$.

Rigid energy. We introduce the rigid energy $E_{r}$ to protect important regions. To achieve protection of important regions, higher importance faces are to be less transformed. This condition can be formulated as

$$
E_{r}=\sum_{f \in \mathbf{F}} s_{f}\left\|\boldsymbol{J}_{f}-\boldsymbol{I}\right\|_{F}^{2}
$$

where $\boldsymbol{I}$ is an identity matrix and $s_{f}$ is an importance of $f$ that is calculated by summing the pixel importance on $f$. $E_{r}$ increases more as a face is further deformed.

Smoothing energy. We introduce the smoothing energy $E_{s}$ to avoid extreme deformation. To achieve smooth transformation, the transformation of a face should be as similar as possible to the transformation of its adjacent faces. This condition can be formulated as

$$
E_{s}=\sum_{f_{i} \in \mathbf{F}} \sum_{f_{j} \in N\left(f_{i}\right)}\left(a_{f_{i}}+a_{f_{j}}\right)\left\|\boldsymbol{J}_{f_{i}}-\boldsymbol{J}_{f_{j}}\right\|_{F}^{2}
$$

where $N\left(f_{i}\right)$ is a set of adjacent faces that share an edge with $f_{i}$. $E_{s}$ increases more with an increasing difference in the transformation of a face between its adjacent faces.

Cropping energy. Our proposed method imposes a constraint to vertex positions in order to incorporate our warping approach with cropping. Let $\Psi$ and $\mathbf{V}(\Psi)$ be a rectangular range not to be discarded and a set of all vertices inside $\Psi$, respectively. Our method adds the following constraint to the energy minimization problem in order to guarantee that all the vertices of $\mathbf{V}(\Psi)$ are inside the target range:

$$
0 \leq v_{i, x}^{\prime} \leq W^{\prime}, 0 \leq v_{i, y}^{\prime} \leq H^{\prime} \quad \text { if } \quad \boldsymbol{v}_{i} \in \mathbf{V}(\Psi)
$$

In addition, we add the following constraints in order to 
guarantee that the transformed mesh completely covers the target range:

$$
\left\{\begin{array}{lll}
v_{i, x}^{\prime} \leq 0 & \text { if } & v_{i, x}=0, \\
v_{i, x}^{\prime} \geq W^{\prime} & \text { if } & v_{i, x}=W, \\
v_{i, y}^{\prime} \leq 0 & \text { if } & v_{i, y}=0, \\
v_{i, y}^{\prime} \geq H^{\prime} & \text { if } & v_{i, y}=H .
\end{array}\right.
$$

Using the hard constraint in Eq. (10) is similar to [9]. This approach enables our method to apply cropping prior to the deformation of the faces of the mesh when an image width is reduced. $E_{a 1}, E_{a 2}, E_{r}$ and $E_{s}$ are obviously zero when all faces are untransformed, i.e., $\boldsymbol{J}_{f}=\boldsymbol{I}$ for all $f$. Thus, only cropping is applied when all faces can be untransformed in image reduction. This is preferable because cropping is able to produce artifact-free results in principle. However, this method fails to uniquely determine the range to be cropped if the target width $W^{\prime}$ is greater than the width of the rectangular range $\Psi$ when an image width is reduced. For example, when using energies defined above, different results of cropping in Fig. 3 (c), (d) and (e) have the same minimum energy, i.e., zero.

We introduce the cropping energy $E_{c}$ to specify the range to be cropped. Our method focuses on the centroid of image importance. In the source image of Fig. 3, visual importance is mainly located at the center of the image. If this composition is intentional for a photographer, it is preferable to preserve the location of visual importance in order to protect one's idea. Therefore, our method imposes the range to be cropped to preserve the importance centroid normalized by the image size as much as possible in the retargeting. Figure 3 (e) is an example of the preservation of the importance centroid.

The centroid of image importance can be approximated by the following weighted average of the centroids of all the faces:

$$
\boldsymbol{g}_{\mathbf{F}}=\frac{1}{\sum_{f \in \mathbf{F}} s_{f}} \sum_{f \in \mathbf{F}} s_{f} \boldsymbol{g}_{f}
$$

We formulate the energy $E_{c}$ for preservation of the normalized centroid of image importance as

$$
E_{c}=\left\|\left[\begin{array}{cc}
1 / W & 0 \\
0 & 1 / H
\end{array}\right] \boldsymbol{g}_{\mathbf{F}}-\left[\begin{array}{cc}
1 / W^{\prime} & 0 \\
0 & 1 / H^{\prime}
\end{array}\right] \boldsymbol{g}_{\mathbf{F}}^{\prime}\right\|_{2}^{2} .
$$

$E_{c}$ increases more with an increasing difference between the normalized centroid of image importance before and after retargeting.

\subsection{Implementation}

Our proposed method first loads an image, and then objects subject to protection of the relative arrangement are manually selected by a user. It may be favorable that objects selected by a user are not to be discarded after retargeting.
Thus, the rectangular range $\Psi$ is set to be a minimum rectangular including all vertices of faces where the objects selected by a user are on. Next, the pixel importance is computed using the saliency detection [10]. This method produces a favorable result with a relatively low computational complexity. Importance values are normalized in the range of $[0,1]$. Finally, an energy minimization problem is solved to obtain an optimal mesh transformation. The energy minimization problem is formulated as follows:

$$
\mathbf{V}_{o p t}^{\prime}=\underset{\mathbf{V}^{\prime}}{\arg \min } \lambda_{a} E_{a}+\lambda_{r} E_{r}+\lambda_{s} E_{s}+\lambda_{c} E_{c}
$$

subject to Eqs. (10) and (11) where $E_{a}=E_{a 1}+E_{a 2}$. This problem is a linear least-squares problem with linear inequality constraints (see Appendix).

\section{Experimental Results and Discussion}

We have implemented our system in MATLAB with Optimization Toolbox to solve the optimization problem. We used a uniform grid mesh divided by $20 \times 20$ faces. Hence, each face is of $W / 20 \times H / 20$ pixels. Weight parameters are set to $\lambda_{a}=20, \lambda_{r}=2, \lambda_{s}=1$, and $\lambda_{c}=1$. The average computation time to retarget an image of size $768 \times 512$ to $384 \times 512$ was 7.1 seconds. We compared our method with Rubinstein's seam carving method [4] and Wang's warping method [5]. In Wang's warping method, each face of a grid mesh is of $20 \times 20$ pixels, which produces sufficiently good results as described in [5]. Our method uses a user input for selection of regions subject to protection of the relative arrangement. To achieve a fair comparison, the pixel importance on the selected regions was set to a maximum value to reduce distortion in the comparison methods [4] and [5].

Figures 4 and 5 represent retargeting results of image reduction. Both our proposed method and the conventional methods protect the shape of the objects. The seam carving method protects the size of an important object as much as possible. Thus, this method reduces unimportant regions among objects, changing the relative arrangement among objects. Wang's warping method allows the change of the size of an important object and protects the shape of the object. Thus, this method reduces less unimportant regions among objects than the seam carving method but fails to protect the relative arrangement among objects. Our method protects the relative arrangement among objects while protecting the shapes of the objects. The protection of the relative arrangement is especially important such as in Fig. 5, where the formation has a significant meaning. Figure 5 (c) and (d) is a comparison without and with the arrangement energy, i.e., $\lambda_{a}=0$ and 20. As shown in this figure, the energy serves to protect the relative arrangement among objects. Figure 6 represents retargeting results of image enlargement. While the conventional methods expand the relative distance between objects, our method appropriately maintains it.

Figure 7 represents retargeting results using quadrilateral and triangle meshes. As can be seen from Fig. 7, our 


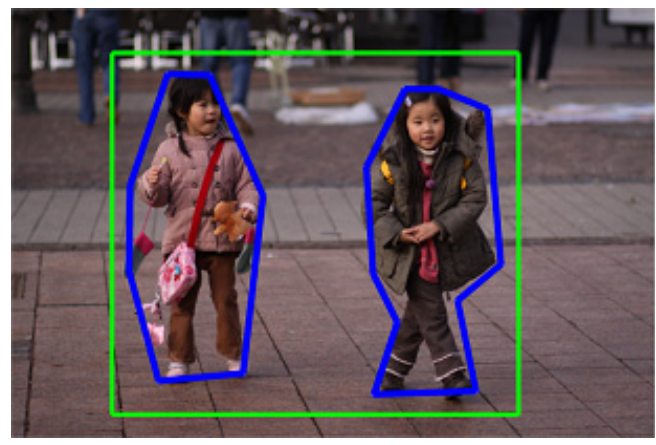

(a)

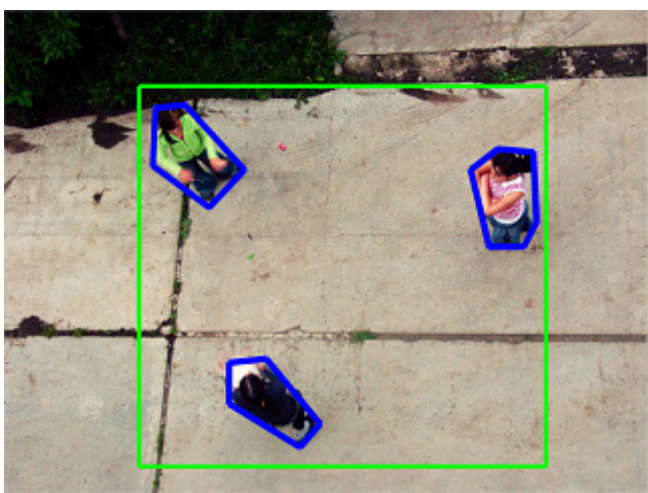

(e)

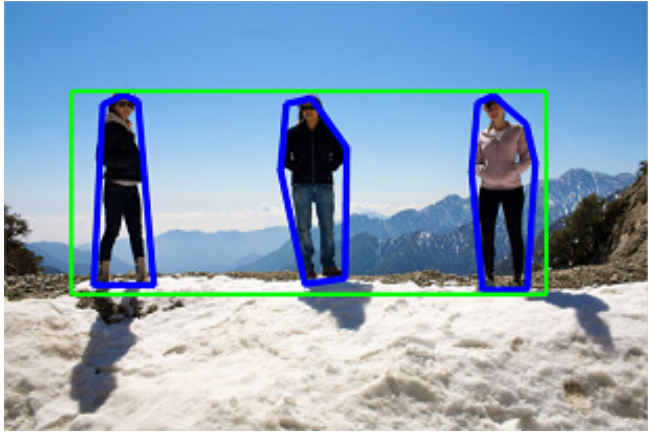

(i)

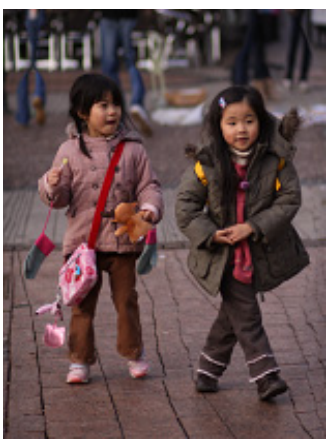

(b)

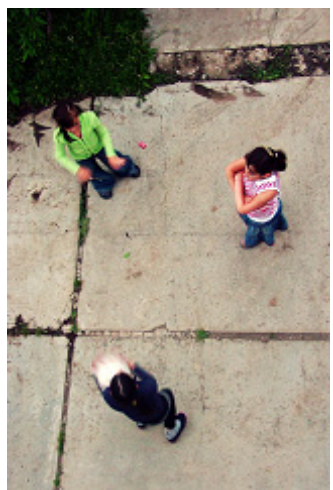

(f)

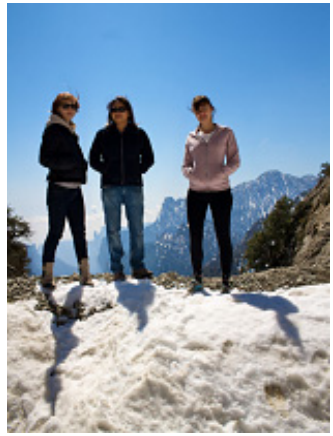

(j)

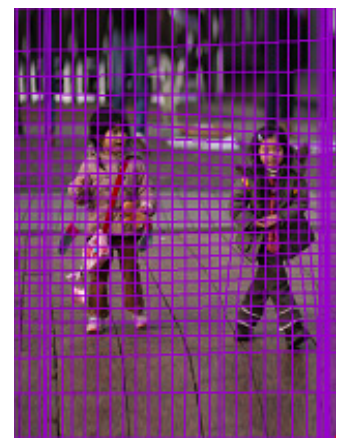

(c)

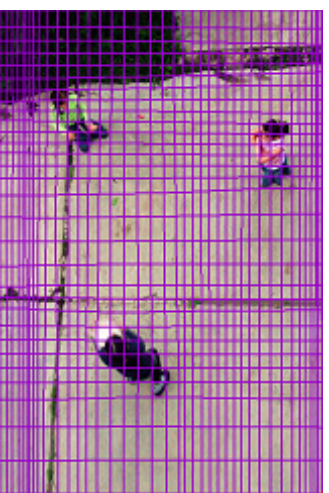

(g)

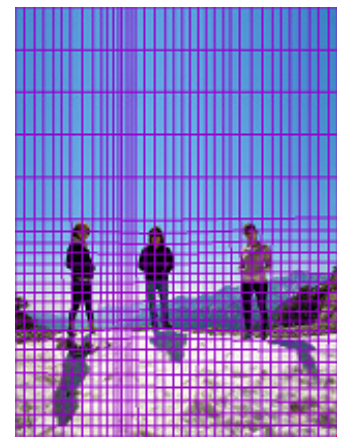

(k)

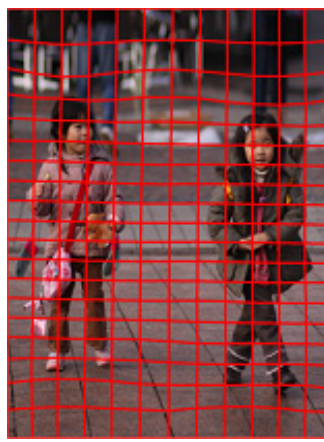

(d)

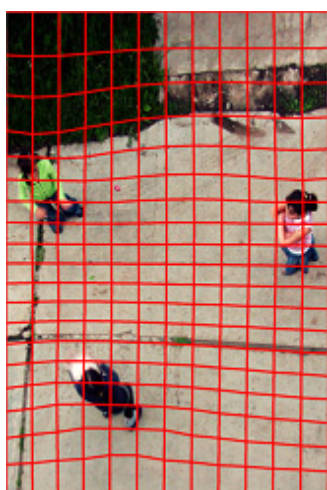

(h)

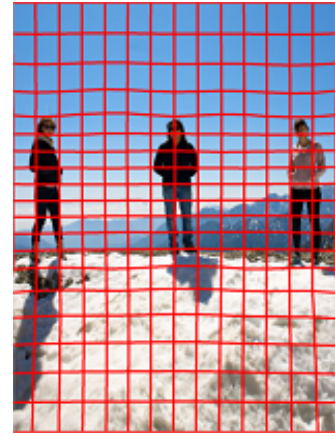

(1)

Fig. 4 Retargeting results of image reduction. (a), (e) and (i) Source images with selected regions (blue) and a rectangular range not to be discarded (green). (b), (f) and (j) Retargeting images using seam carving [4]. (c), (g) and (k) Retargeting images with a deformed mesh using Wang's warping method [5]. (d), (h) and (l) Retargeting images with a deformed mesh using our method.

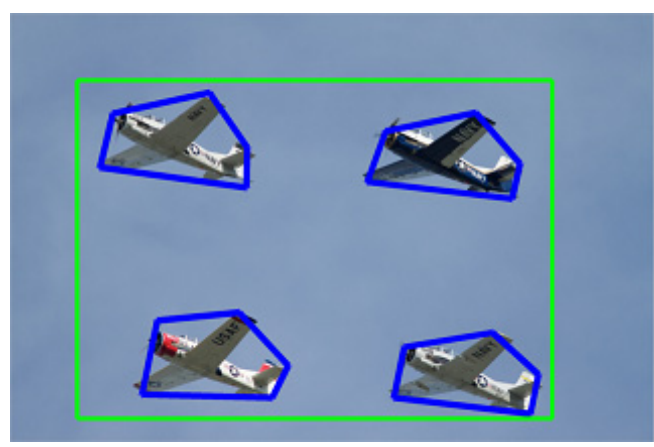

(a)

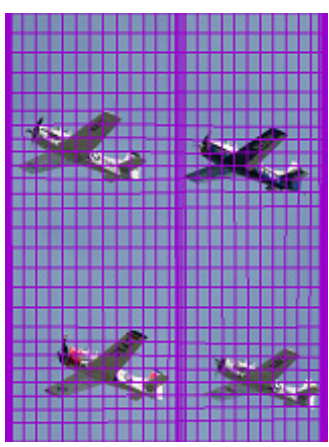

(b)

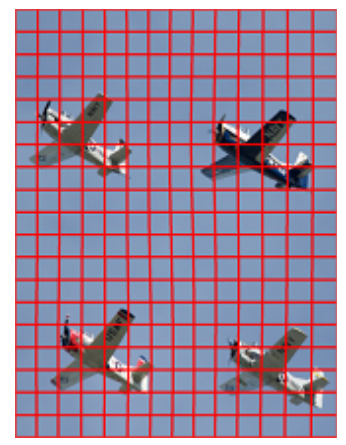

(c)

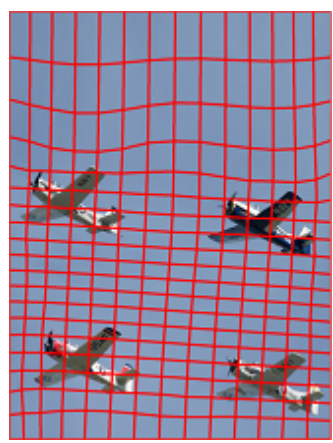

(d)

Fig. 5 Retargeting results of image reduction. (a) Source images with selected regions (blue) and a rectangular range not to be discarded (green). (b) Retargeting image with a deformed mesh using Wang's warping method [5]. (c) and (d) Retargeting image with a deformed mesh using our method without and with the arrangement energy, respectively. 


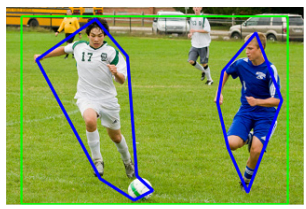

(a)

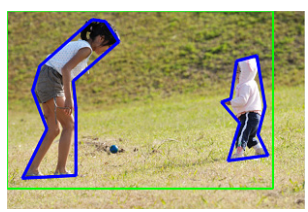

(e)

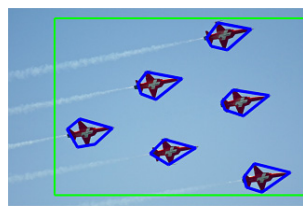

(i)

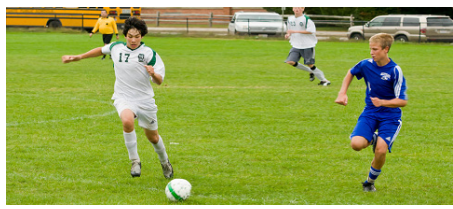

(b)

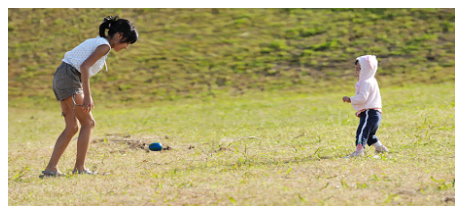

(f)

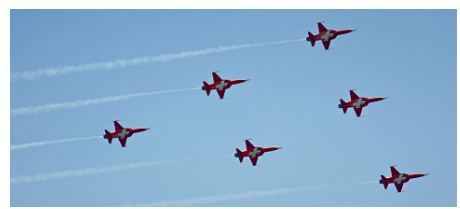

(j)

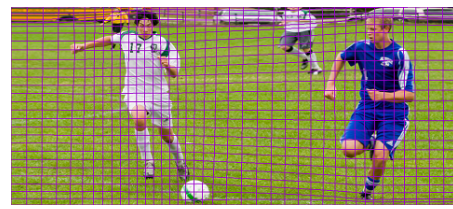

(c)

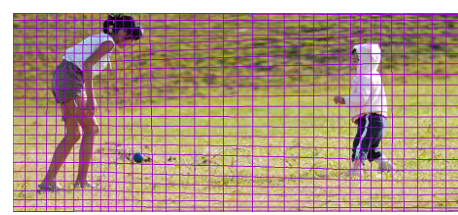

(g)

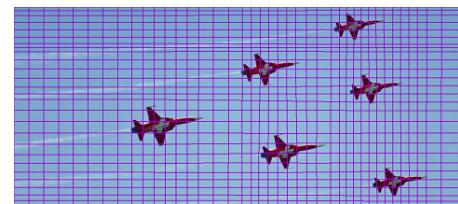

(k)

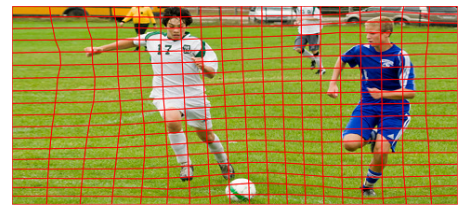

(d)

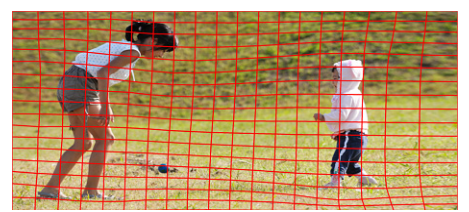

(h)

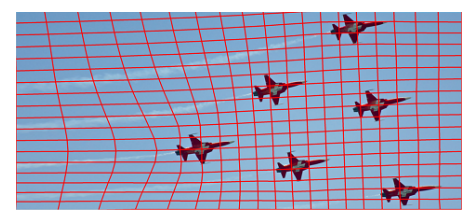

(1)

Fig. 6 Retargeting results of image enlargement. (a), (e) and (i) Source images with selected regions (blue) and a rectangular range not to be discarded (green). (b), (f) and (j) Retargeting images using seam carving [4]. (c), (g) and (k) Retargeting images with a deformed mesh using Wang's warping method [5]. (d), (h) and (l) Retargeting images with a deformed mesh using our method.

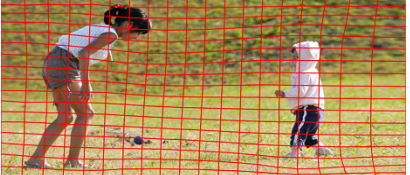

(a)

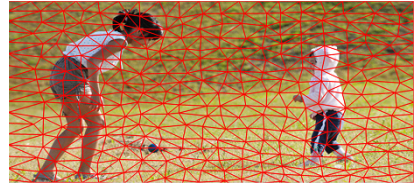

(b)
Fig. 7 Retargeting images with quadrilateral mesh (a) and triangle mesh (b).

energy definition can be used for both types of mesh. In addition, all the energies including the arrangement energy are expressed as a quadratic form, which is commonly used in warping methods. Therefore, the arrangement energy can be incorporated into conventional warping methods.

\section{Conclusions}

We proposed a novel method for retargeting images while maintaining the relative arrangement among objects. To achieve protection of object arrangement, we introduced the arrangement energy that enforces all the objects and the relative positions among these objects to be transformed by the same transformation in the retargeting process. Our method also uses other three types of conditions: protection of important regions, avoiding extreme deformation, and cropping with preservation of the balance of visual importance. Experimental results demonstrate that our proposed method maintains the relative arrangement while protecting important regions.

\section{Acknowledgements}

We thank the following Flickr (http://www.flickr.com/) users for Creative Commons imagery: Stephan Pabst (horse race), gwenael.piaser (two little tourists), shioshvili (old witch), Mike Miley (Formation Flight), B Mlry (Soccer), Todd Ryburn (Birds in flight), melisslissliss (snow mountain), Luis Jou García (Triangle formation). This work was supported by JSPS KAKENHI Grant Number 25820159.

\section{References}

[1] D. Vaquero, M. Turk, K. Pulli, M. Tico, and N. Gelfand, "A survey of image retargeting techniques," Proc. SPIE Applications of Digital Image Processing XXXIII, 2010.

[2] A. Shamir and O. Sorkine, "Visual media retargeting," SIGGRAPH ASIA Courses, New York, NY, USA, pp.1-13, 2009.

[3] S. Avidan and A. Shamir, "Seam carving for content-aware image resizing," ACM Trans. Graph., vol.26, no.3, p.10, 2007.

[4] M. Rubinstein, A. Shamir, and S. Avidan, "Improved seam carving for video retargeting," ACM Trans. Graph., vol.27, no.3, pp.1-9, 2008.

[5] Y.S. Wang, C.L. Tai, O. Sorkine, and T.Y. Lee, "Optimized scaleand-stretch for image resizing," ACM Trans. Graph., vol.27, no.5, pp.118:1-118:8, 2008.

[6] Y. Guo, F. Liu, J. Shi, Z.H. Zhou, and M. Gleicher, "Image retargeting using mesh parametrization," IEEE Trans. Multi., vol.11, no.5, pp.856-867, 2009.

[7] W. Dong, N. Zhou, J. Paul, and X. Zhang, "Optimized image resizing using seam carving and scaling," ACM Trans. Graph., vol.28, no.5, pp.1-10, 2009.

[8] M. Rubinstein, A. Shamir, and S. Avidan, "Multi-operator media retargeting," ACM Trans. Graph., vol.28, no.3, pp.1-11, 2009.

[9] Y.S. Wang, H.C. Lin, O. Sorkine, and T.Y. Lee, "Motion-based video retargeting with optimized crop-and-warp," ACM Trans. Graph., 
vol.29, no.4, pp.90:1-90:9, July 2010.

[10] X. Hou and L. Zhang, "Saliency detection: A spectral residual approach," Proc. IEEE Conf. Computer Vision and Pattern Recognition, pp.1-8, 2007.

\section{Appendix: Optimization problem}

$E_{a 1}$ and $E_{a 2}$ can respectively be transformed into the following:

$$
\begin{aligned}
& E_{a 1}=\sum_{\omega \in \Omega} \sum_{f \in \mathbf{F}(\omega)} a_{f}\left\|\left[\begin{array}{cc}
1 & 0 \\
0 & -1 \\
0 & 1 \\
1 & 0
\end{array}\right] \boldsymbol{t}-\operatorname{vec}\left(\boldsymbol{J}_{f}\right)\right\|_{2}^{2}, \\
& E_{a 2}=\sum_{\left(\omega_{i}, \omega_{j}\right) \in \Phi_{\Omega}} l_{i, j}\left\|\left[\begin{array}{cc}
g_{x} & -g_{y} \\
g_{y} & g_{x}
\end{array}\right] \boldsymbol{t}-\left[\begin{array}{l}
g_{x}^{\prime} \\
g_{y}^{\prime}
\end{array}\right]\right\|_{2}^{2}
\end{aligned}
$$

where $\boldsymbol{t}=[c, d]^{T}, \boldsymbol{g}_{\omega_{i}, \omega_{j}}=\left[g_{x}, g_{y}\right]^{T}$, and vec(.) is an operator that creates a column vector from a matrix by stacking the column vectors of the matrix. Since vec $\left(\boldsymbol{J}_{f}\right)$ and $\boldsymbol{g}_{\omega_{i}, \omega_{j}}$ are represented as a linear combination of the vertex positions after retargeting $\boldsymbol{x}=\left[v_{1, x}^{\prime}, \ldots, v_{\alpha, x}^{\prime}, v_{1, y}^{\prime}, \ldots, v_{\alpha, y}^{\prime}\right]^{T}, E_{a}$ can be written as the following quadratic form in $\boldsymbol{x}$ :

$$
\|\boldsymbol{B} \boldsymbol{t}-\boldsymbol{A x}\|_{2}^{2}
$$

The optimal $\boldsymbol{t}$ can be found by solving $\boldsymbol{A x}=\boldsymbol{B} \boldsymbol{t}$ in a least square sense: $\boldsymbol{t}=\boldsymbol{B}^{+} \boldsymbol{A} \boldsymbol{x}$. Thus, $E_{a}$ can be written as follows:

$$
\left\|\left(\boldsymbol{I}-\boldsymbol{B} \boldsymbol{B}^{+}\right) \boldsymbol{A} \boldsymbol{x}\right\|_{2}^{2} .
$$

Since $E_{r}, E_{s}$ and $E_{c}$ are also quadratic with respect to the vertex positions after retargeting, Eq. (14) subject to Eqs. (10) and (11) is a linear least-squares problem with linear inequality constraints.

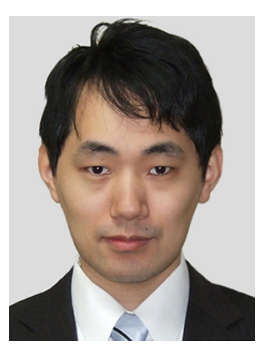

Kazu Mishiba received the B.E. and M.E. in 2004 and 2006, respectively from Keio University, Yokohama, Japan. In 2006, He joined FUJI FILM CO., Ltd. He received the Ph.D. in 2011 from Keio University. He was an assistant professor at Keio University in 2011. He is currently an assistant professor at Tottori University. His research interests are in the areas of image interpolation, content-aware resizing, and image editing.

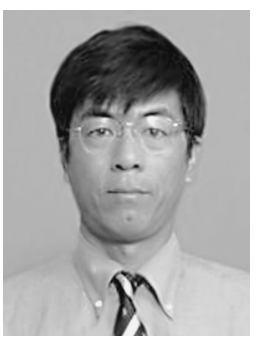

Takeshi Yoshitome

received the B.S. and M.S. degrees in computer science from Tsukuba University, Japan, in 1982 and 1984, respectively. In 1984, he joined the Electrical Communication Laboratories, Nippon Telegraph and Telephone Corporation (NTT), Kanagawa, Japan and has been engaged in research and development of image processing systems. He received the Ph.D. degree in computer science from Tsukuba University in 2010. He is currently a professor at Tottori University. 